\title{
Ultrastructural changes of oocyte and follicular wall during oocyte maturation in the Japanese quail (Coturnix coturnix japonica)
}

\author{
Y. Yoshimura, T. Okamoto and T. Tamura \\ Faculty of Applied Biological Science, Hiroshima University, Higashi-Hiroshima 724, Japan
}

\begin{abstract}
This study examined structural changes in oocyte and follicular wall during oocyte maturation in Japanese quails. The structures of the germinal disc and the surrounding follicular wall were observed by light and electron microscopy 25, 6 and $1 \mathrm{~h}$ before the expected time of ovulation. The germinal disc of the oocyte was located near the oocyte plasma membrane at $25 \mathrm{~h}$ before ovulation, and the germinal vesicle was located in the centre of the germinal disc. Numerous cytoplasmic elements, such as elongated membrane-bound vesicles, mitochondria and glycogen granules were also observed in the germinal disc. The surface of the oocyte made close contact with the cytoplasmic processes of the granulosa cells. Six hours before ovulation, fluid filled spaces formed between the oocyte and follicular wall. At $6 \mathrm{~h}$ before ovulation, the germinal disc was similar to that at $25 \mathrm{~h}$ before ovulation, whereas the oocyte and the granulosa cells were disconnected. Myelin bodies and dense bodies developed in the cytoplasmic processes of the granulosa cells, suggesting that lysosomal enzymes were activated. In the follicle at $\mathrm{I} h$ before ovulation, the second maturation spindle was located just beneath the surface of the oocyte, and the first polar body was in the perivitelline space. In the germinal disc, the membrane-bound vesicles were swollen and well developed. We suggest that, during the process of early oocyte maturation, the junctions between the oocyte and granulosa cells are disconnected, and factors that promote oocyte maturation may be activated in the germinal disc since the membrane-bound vesicles are developed.
\end{abstract}

\section{Introduction}

In birds, the germinal disc, a thickened mass of protoplasm of the oocyte, is located in the periphery of the oocyte. The germinal vesicle lies in the centre of the germinal disc. It has been suggested that germinal vesicle breakdown is induced by luteinizing hormone $(\mathrm{LH})$, since germinal vesicle breakdown occurs immediately after the LH surge (approximately 4-6 h before ovulation) in normally laying birds and germinal vesicle breakdown is induced by an injection of the pituitary extracts (Olsen and Fraps, 1950). The maturation of the oocyte is also induced by LH or human chorionic gonadotrophin (hCG) in mammals (Lindner et al., 1974; Tsafriri et al., 1982) and lower vertebrates (Patino and Thomas, 1990).

More precise mechanisms of oocyte maturation have been elucidated. A factor produced by the granulosa cells prevents the maturation of the oocyte in immature mammalian follicles (Sato and Koide, 1984; Anderson et al., 1985). This factor does not prevent the maturation of the oocyte in mature follicles because the communication between the oocyte and the granulosa cells is removed and the factor is not taken up by the oocyte (Sirard and Bilodean, 1990). Moreover, progesterone or the other steroid hormones produced by the granulosa cells under the control of LH may induce oocyte maturation through receptors on the oocyte plasma membrane in lower vertebrates

Received 14 February 1992.
(Haider, 1990). In the cytoplasm of the immature oocyte, maturation-promoting factor (MPF), a metaphase regulating factor, occurs in an inactive form. The maturation-promoting factor of lower vertebrates is activated by progesterone or other steroid hormones and plays an essential role in oocyte maturation (Haider, 1990; Motlik and Kubelka, 1990). MPF has also been reported in the avian oocyte (Mori et al., 1991).

Olsen (1942) and Olsen and Fraps (1950), using light microscopy, reported that, in hens, the germinal vesicle wall appears to weaken and eventually ruptures approximately $6 \mathrm{~h}$ before ovulation, allowing the matrix of the vesicle to spread laterally as a thin sheet. They also observed that condensation of chromatin, extrusion of the first polar body and formation of the second maturation spindle are completed $2.5 \mathrm{~h}$ before ovulation. However, more detailed histological data are required to confirm the significance of the results of recent physiological experiments on oocyte maturation. The ultrastructural changes of the oocyte and follicular wall during the process of oocyte maturation were observed in this study. Specifically, changes of the oocyte and follicular wall junctions and changes of the germinal disc structures were observed.

\section{Materials and Methods}

Sixteen female Japanese quails that had laid five or more eggs in a sequence were maintained in individual cages under a 
regimen of $14 \mathrm{~h}$ light (from 6:00 to $20: 00 \mathrm{~h}$ ) and $10 \mathrm{~h}$ dark, and provided with food and water ad libitum. They were laying the first or second eggs in a clutch between 16:00 to 18:00 h. Ovulation occurs approximately $30 \mathrm{~min}$ after oviposition in hens (Phillips and Warren, 1937). The birds were killed by decapitation. Follicles were collected $25 \mathrm{~h}, 6 \mathrm{~h}$ and $\mathrm{th}$ before the expected time of ovulation. The follicles were fixed in Karnovsky's fixative (4\% paraformaldehyde and 5\% glutaraldehyde in Millonig's buffer, consisting of $0.12 \mathrm{~mol} \mathrm{NaH}_{2} \mathrm{PO}_{4} \mathrm{l}^{-\mathrm{I}}$ and $0.12 \mathrm{~mol} \mathrm{NaOH} \mathrm{l}^{-1} \mathrm{pH} \mathrm{7.2)}$ at $4^{\circ} \mathrm{C}$ for $1 \mathrm{~h}$. The loose connective tissue and superficial epithelium covering the follicular wall were then removed with forceps. The germinal disc region of the oocyte with the follicular wall was cut out and fixed over night in the same fixative at $4{ }^{\circ} \mathrm{C}$. After washing with Millonig's buffer for $2 \mathrm{~h}$, the tissues were postfixed in $1.3 \% \mathrm{OsO}_{4}$ in Millonig's buffer at $4^{\circ} \mathrm{C}$ for $2 \mathrm{~h}$. They were dehydrated with acetone and embedded in Epon 812. Thick sections stained with toluidine blue were observed under a light microscope. Thin sections were stained with uranyl acetate and lead acetate and examined under JEM 1200 EX electron microscope at an accelerating voltage of $60 \mathrm{KV}$.

\section{Results}

\section{Light microscope observations}

At $25 \mathrm{~h}$ before ovulation the germinal vesicle was in the centre of the germinal disc and contained weakly stained nuclear matrix (Fig. 1a). The apical surface of the germinal vesicle was flattened against the surface of the oocyte, and the basal surface of it was convex in shape. The follicular wall consisted of the perivitelline layer, granulosa layer, theca interna and theca externa from inside to outside (Fig. 1b). The perivitelline layer was densely stained and located between the oocyte and the granulosa layer. The granulosa layer was a simple epithelium of cuboidal cells. The theca interna consisted of connective tissue with fibroblasts and thecal interstitial cells, and capillary sinus (Nishida et al., 1977) distributed throughout the layer.

In the follicles collected $6 \mathrm{~h}$ before the expected time of ovulation, six of 11 oocytes showed the early structural changes associated with oocyte maturation (Fig. 2a), whereas five oocytes did not show significant structural changes. In the follicles in which the oocyte was undergoing maturation, spaces were formed between the oocyte surface and perivitelline layer on the germinal disc. Lightly stained fluid accumulated in these spaces. The intercellular spaces of the granulosa cells were widened in the area of the germinal disc region of these follicles (Fig. 2b). Furthermore, the theca interna appeared loosened (Fig. $2 \mathrm{~b}$ ) compared with the theca interna from follicles collected $25 \mathrm{~h}$ before ovulation (Fig. 1b). By contrast, the germinal vesicle (Fig. 2a) still contained, in its central part, spherical chromosomes typical of the postlampbrush chromosome stage (Callebaut, 1973).

One hour before ovulation the oocyte was typical of metaphase II. A second maturation spindle which had the dense chromosomes lying in an equatorial band was located just beneath the surface of the oocyte (Fig. 3a). The perivitelline space was formed between the surface of the germinal disc and the perivitelline layer. The first polar body composed of a small amount of matrix and chromatin was located in the perivitelline space (Fig. 3b). The intercellular spaces between the granulosa cells above the germinal disc were not as wide as those seen in the follicle granulosa collected $6 \mathrm{~h}$ before ovulation.

\section{Electron microscope observations}

Electron micrographs revealed that in the follicle at $25 \mathrm{~h}$ before ovulation numerous oocyte cytoplasmic elements such as mitochondria, smooth membrane-bound vesicles and glycogen granules were located in the peripheral region of the germinal vesicle (Fig. 4a). This zone corresponds to the localization of the perinuclear halo of ticos $-\left[{ }^{3} \mathrm{H}\right]$ thymidine incorporated cytoplasmic organelles described by Callebaut (1983, 1987). These ooplasmic elements were also distributed in a narrow space between the germinal vesicle and the surface of the oocyte. The germinal vesicle consisted of the distinct inner and outer nuclear membrane and nuclear matrix (Fig. 4b). In the germinal disc region, distant from the germinal vesicle, elongated smooth membrane-bound vesicles were distributed immediately beneath the oocyte plasma membrane and yolk spheres of approximately $1 \mu \mathrm{m}$ diameter accumulated under the vesicles. The surface of the germinal disc was irregular and the oocyte plasma membrane formed the microvilli. The cytoplasmic process of the granulosa cells protruded through the perivitelline layer and extended to the oocyte surface. The microvilli of the oocyte interdigitated with the cytoplasmic process of the granulosa cells (Fig. 5). Moreover, the cytoplasmic process of the granulosa cells formed the junctional structure of spot desmosome with the oocyte plasma membrane (inset of Fig. 5). The granulosa cells contained numerous cytoplasmic organelles such as oval mitochondria, rough endoplasmic reticulum and Golgi apparatus. The granulosa cells were generally separated by a narrow space, and a few regions of their lateral wall were attached closely to the adjacent cells (Figs 4b, 5).

At the early stage of oocyte maturation, $6 \mathrm{~h}$ before the expected time of ovulation, the germinal vesicle and the other cytoplasmic organelles of the oocyte did not show significant structural changes (Fig. 6). However, the junctions between the microvilli of the oocyte and cytoplasmic process of the granulosa cells were disconnected from each other. Dense bodies and myelin bodies developed in the cytoplasmic processes of the granulosa cells (Fig. 7). The inner structure of the granulosa cells were not significantly different (Fig. 7) from those of the follicles $25 \mathrm{~h}$ before ovuation (Fig. $4 \mathrm{~b}$ ).

In the germinal disc of the oocyte $1 \mathrm{~h}$ before the expected time of ovulation, numerous membrane-bound vesicles of various sizes were distributed beneath the plasma membrane (Fig. 8). These vesicles were apparently the swollen structures of the elongated membrane-bound vesicles observed in the germinal disc $25 \mathrm{~h}$ before ovulation (Fig. 5). The microvilli of the oocyte were erect and disconnected from the cytoplasmic process of the granulosa cells.

\section{Discussion}

This study showed (i) that the oocyte plasma membrane formed junctional structures with the cytoplasmic processes of the 



Fig. 1. (a) Light micrograph of a cross-section of the germinal disc $25 \mathrm{~h}$ before ovulation. The germinal vesicle (GV) is located in the centre of the germinal disc (GD) which is surrounded by a follicular wall (FW). Scale bar $=100 \mu \mathrm{m}$. (b) Light micrograph of the same germinal disc. The follicular wall consists of the granulosa layer $(\mathrm{G})$, theca interna (TI) and theca externa (TE). Arrow: perivitelline layer; GV: germinal vesicle. Scale bar $=20 \mu \mathrm{m}$.
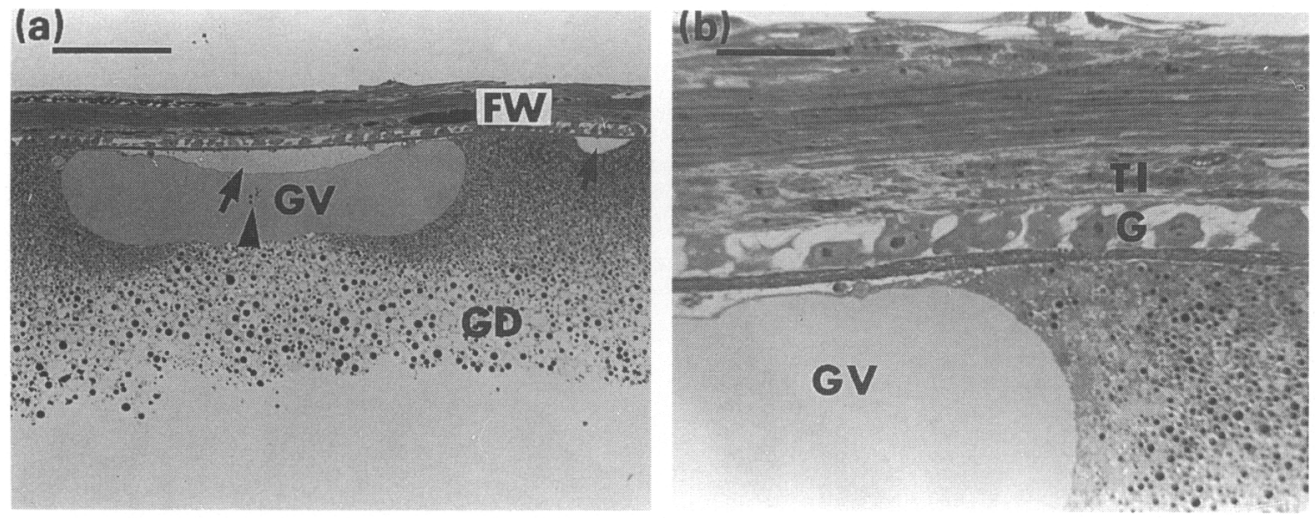

Fig. 2. (a) Light micrograph of a cross-section of the germinal disc $6 \mathrm{~h}$ before ovulation. Note that spaces (arrows) are formed between the surface of the oocyte and the perivitelline layer. The fluid in the space is stained very lightly. FW: follicular wall; GD: germinal disc; GV: germinal vesicle; within its central part are spherical postlampbrush chromosomes (arrowhead). Scale bar $=100 \mu \mathrm{m}$. (b) Light micrograph of the same germinal disc. The intercellular space of the granulosa layer $(G)$ is widened and the theca interna (TI) is loosened compared with the same structures $25 \mathrm{~h}$ before ovulation (Fig. 1b). GV: germinal vesicle. Scale bar $=20 \mu \mathrm{m}$.
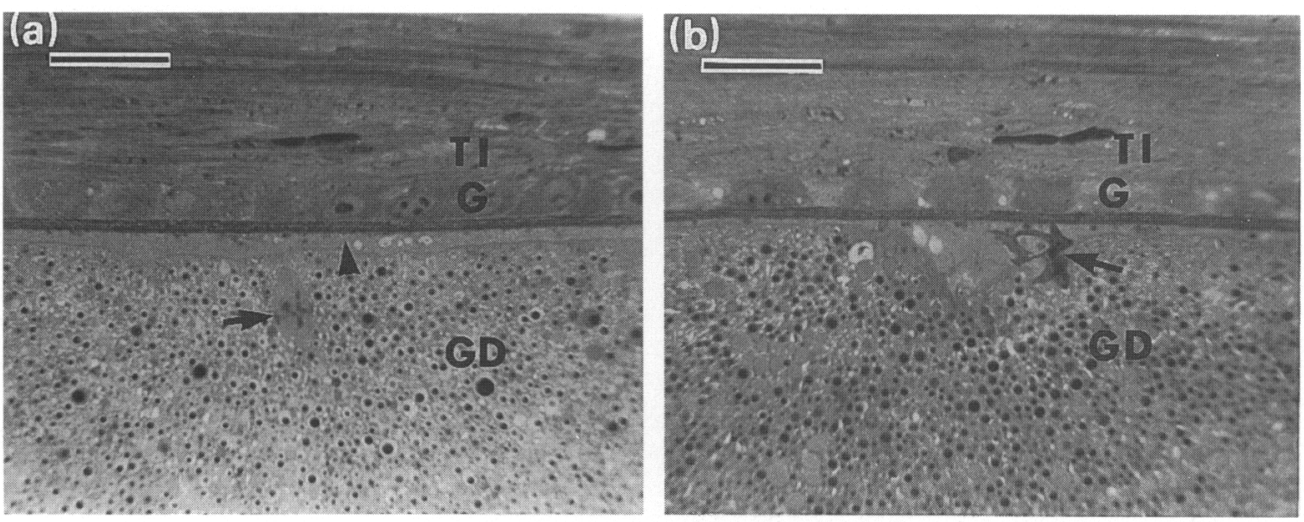

Fig. 3. (a) Light micrograph of a cross-section of the germinal disc $1 \mathrm{~h}$ before ovulation. The second maturation spindle which contains chromosomes is seen (arrow). Arrowhead: perivitelline space; G: granulosa layer; GD: germinal disc; TI: theca interna. Scale bar $=20 \mu \mathrm{m}$. (b) Light micrograph of the same germinal disc. The first polar body is located in the perivitelline space (arrow). G: granulosa layer; GD: germinal disc; TI: theca interna. Scale bar $=20 \mu \mathrm{m}$. 



Fig. 4. (a) Electron micrograph of the germinal disc $25 \mathrm{~h}$ before ovulation. Numerous organelles and glycogen granules $(G)$ are distributed in the germinal disc near the germinal vesicle (GV). M: mitochondria; PV: perivitelline layer; V: membrane-bound vesicle; $Y$ : yolk sphere. Scale bar $=1.4 \mu \mathrm{m}$. (b) Electron micrograph of the germinal disc $25 \mathrm{~h}$ before ovulation. The narrow space between the germinal vesicle $(\mathrm{GV})$ and oocyte plasma membrane is occupied by ooplasm (arrow). The surface of the oocyte contacts with the perivitelline layer (PV). BM: basement membrane; GC: granulosa cell. Scale bar $=1.4 \mu \mathrm{m}$. Inset shows the magnified view of the oocyte-follicular wall border. Arrow: cytoplasmic process of the granulosa cell; arrowhead: oocyte plasma membrane; double arrows: nuclear membrane of germinal vesicle. Scale bar $=240 \mathrm{~nm}$.

granulosa cells in the immature follicles, whereas these junctions disintegrated during the early stage of maturation; (ii) that the fluid accumulated between the oocyte surface and perivitelline layer during the early stage of maturation; and (iii) that smooth membrane-bound vesicles in the ooplasm developed during maturation.

The formation of the space between the oocyte surface and perivitelline layer followed by the disintegration of the 


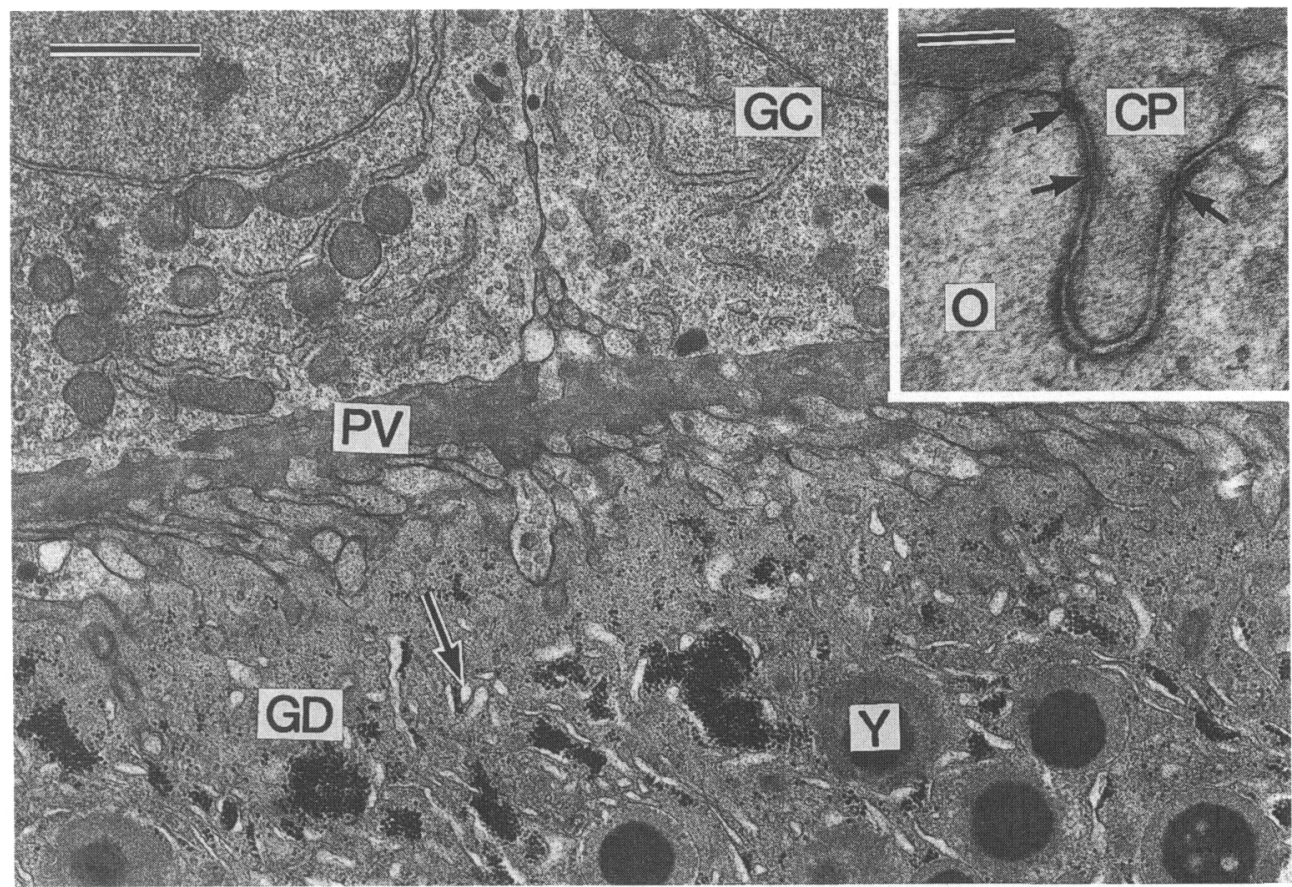

Fig. 5. Electron micrograph of the germinal disc distant from the germinal vesicle $25 \mathrm{~h}$ before ovulation. Cytoplasmic processes of the granulosa cells interdigitate with the oocyte microvilli under the perivitelline layer (PV). Note that the elongated smooth membrane-bound vesicles are distributed (arrow). GC: granulosa cell; GD: germinal disc; Y: yolk sphere. Scale bar $=1.4 \mu \mathrm{m}$. Inset shows the junctions of the cytoplasmic process of the granulosa cell (CP) with oocyte plasma membrane. Arrows: spot desmosome; O: oocyte. Scale bar $=240 \mathrm{~nm}$.

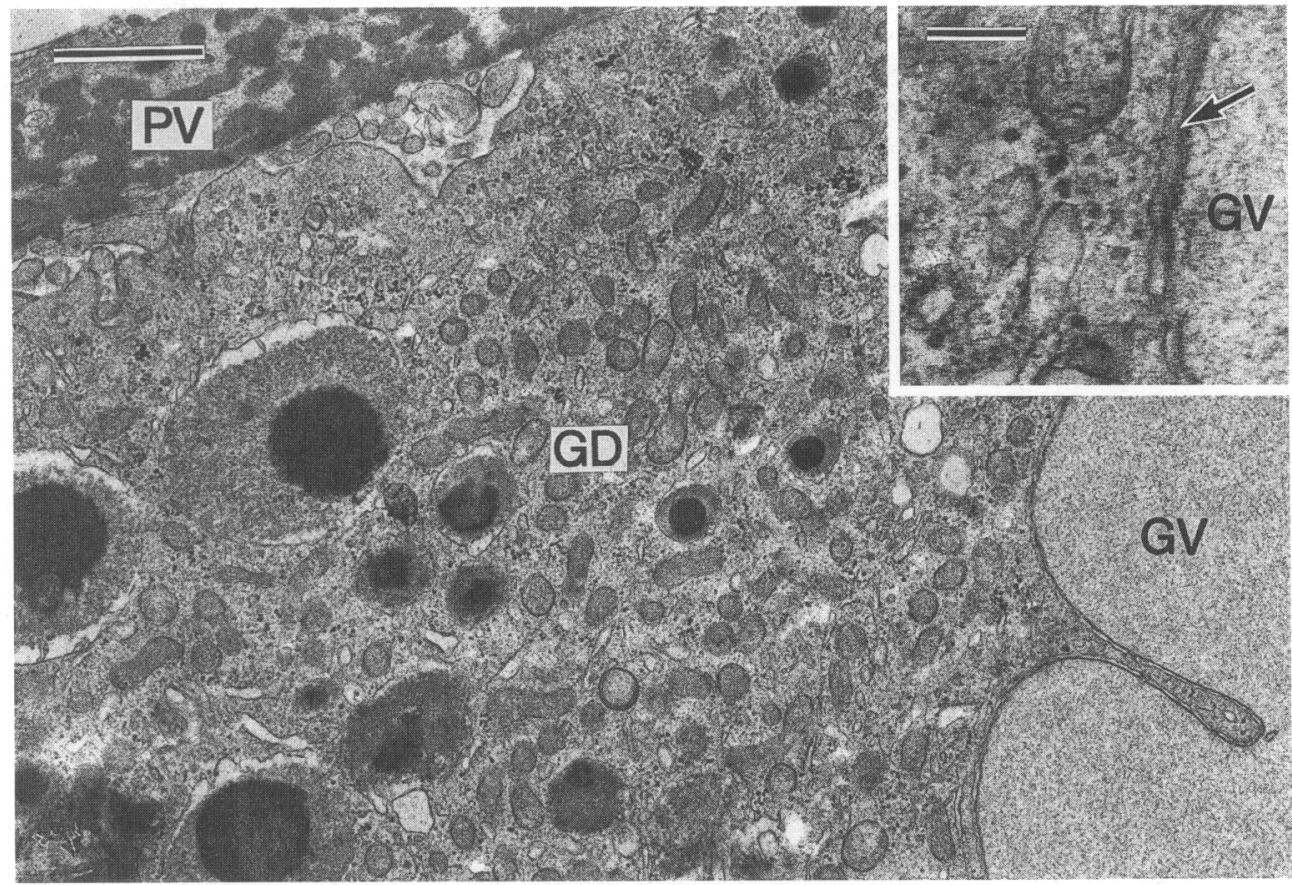

Fig. 6. Electron micrograph of the germinal disc $6 \mathrm{~h}$ before ovulation. Numerous cytoplasmic organelles distribute near the germinal vesicle (GV) in a same manner as in the germinal disc $25 \mathrm{~h}$ before ovulation (Fig. 4a). GD: germinal disc; PV: perivitelline layer. Scale bar $=1.4 \mu \mathrm{m}$. Inset shows that the nuclear membrane of the germinal vesicle (GV) is still intact (arrow). Scale bar $=240 \mathrm{~nm}$. The accumulation of round mitochondria in the angle between the germinal vesicle and surface of the ooplasm suggests the presence of tico $\left({ }^{3} \mathrm{H}\right]$ thymidine-incorporating cytoplasmic organelle) materials. 




Fig. 7. Electron micrograph of the germinal disc $6 \mathrm{~h}$ before ovulation. A wide space is formed between the surface of the germinal disc and the perivitelline layer (PV). Note the myelin body and dense bodies in the cytoplasmic process of the granulosa cell (arrow). GC: granulosa cell; GV: germinal vesicle. Scale bar $=1.4 \mu \mathrm{m}$.

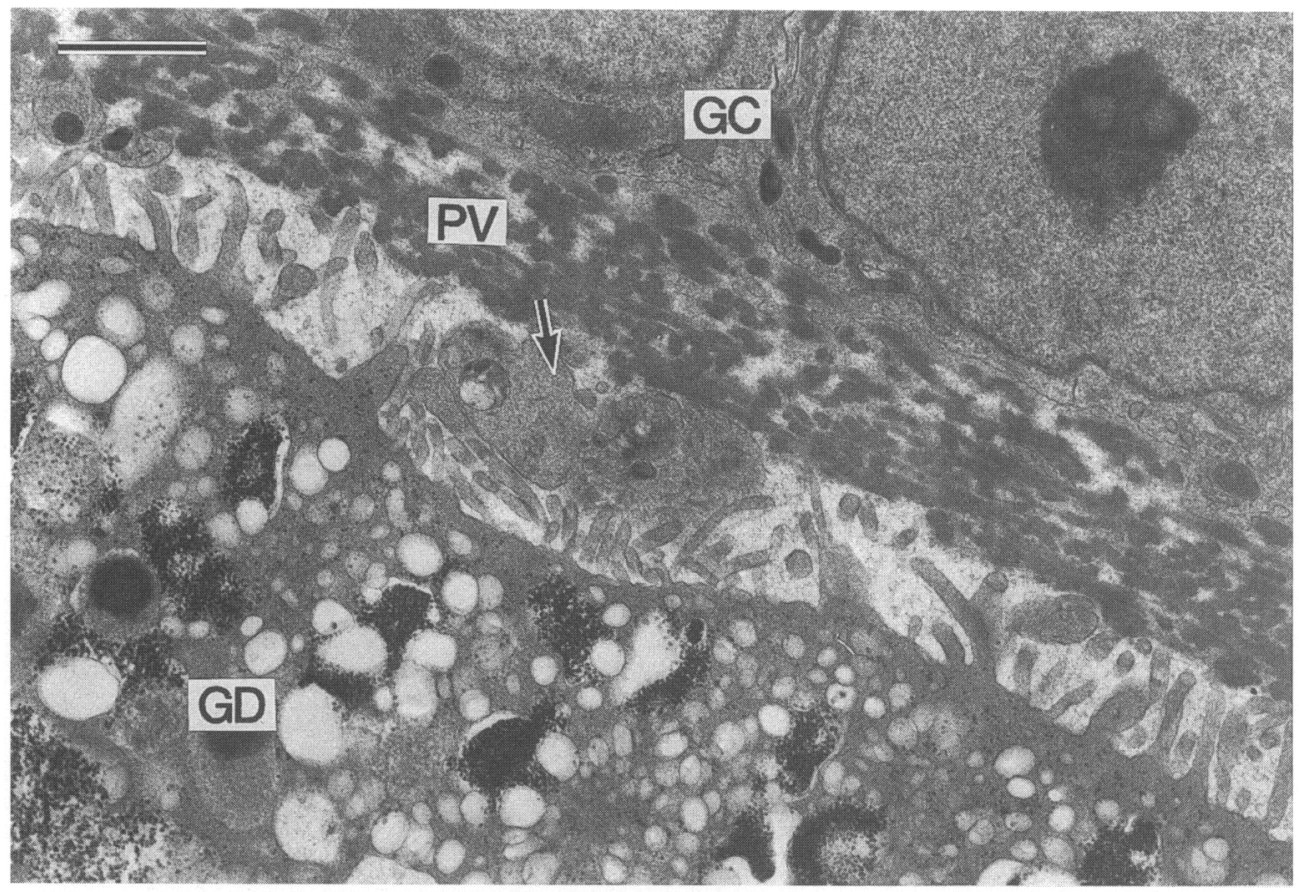

Fig. 8. Electron micrograph of the germinal disc $\mathrm{I}$ h before ovulation. Note the numerous swollen membrane-bound vesicles in the germinal disc (GD). Arrow indicates the cytoplasmic processes of the granulosa cells. GC: granulosa cell; PV: perivitelline layer. Scale bar $=1.4 \mu \mathrm{m}$.

junctional structures between the oocyte and the granulosa cells were the first indication of oocyte maturation. These structural changes were observed in six of the 11 follicles collected $6 \mathrm{~h}$ before the expected time of ovulation. The $\mathrm{LH}$ surge occurs
4-6 h before ovulation in quails and chickens (Doi et al., 1980; Johnson and van Tienhoven, 1980). It is, therefore, considered that the first indications of oocyte maturation occur after the LH surge. The reason why five of the 1]. follicles did not show 
significant structural changes may be that the time from the $\mathrm{LH}$ surge to sample collection was too short and the effects of $\mathrm{LH}$ had not been expressed. One hour before the expected time of ovulation, the first polar body of all follicles was located in the perivitelline space and the second maturation spindle was located just beneath the surface of the germinal disc. These results support those of Olsen and Fraps (1950), who reported that the onset of maturation changes occurred approximately $4.5 \mathrm{~h}$ before ovulation and extrusion of the first polar body $2.5 \mathrm{~h}$ before ovulation, in the chicken.

This is the first report that clearly shows that the junctions between the oocyte and the granulosa cells disintegrate during the process of the oocyte maturation in quail hens. Before the maturational changes are initiated in the oocyte, the cytoplasmic processes of the granulosa cells were interdigitated with the oocyte microvilli and formed the spot desmosome with the oocyte plasma membrane. These interdigitations and spot desmosomes between the oocyte and the granulosa cells have been described in chicken follicles (Perry et al., 1978a, b). The disintegration of the junctions between the oocyte and the granulosa cells occurred $6 \mathrm{~h}$ before the expected time of ovulation, i.e. just after the LH surge and just before germinal vesicle breakdown. We suggest that two different factors induce the disintegration of the junctions between the oocyte and granulosa cells, namely enzymatic digestion and the contraction of the granulosa cells. At the time of disintegration, dense bodies and myelin bodies were present in the cytoplasmic process of the granulosa cells, suggesting that the lysosomal enzymes were activated in this region. Moreover, it has been indicated that chicken granulosa cells produce plasminogen activator (Tilly and Johnson, 1987). These enzymes may therefore be responsible for the disintegration of the oocyte and granulosa cell junctions. On the other hand, the granulosa cells showed a contracted appearance, forming a wide intercellular space. We have shown that cyclic AMP induces the contraction of cultured granulosa cells (Yoshimura and Tamura, 1991). The granulosa cells of the FI follicle produce a large amount of cyclic cAMP in response to $\mathrm{LH}$ (Calvo et al., 1981). It is therefore anticipated that the contraction of the granulosa cells is induced by cyclic cAMP and the disintegration of the junctions between the oocyte and granulosa cells is promoted.

The physiological significance of the disintegration of the junctions between the oocyte and the granulosa cells remains to be investigated in hens. There is no evidence for gap junctions between the immature oocyte and granulosa cells in birds. However, in mammals, it is reported that the oocyte forms the gap junctions with the surrounding cumulus cells (Gilula et al., 1978), and the junctions are disconnected during oocyte maturation (Hyttel et al., 1986). Moreover, it is suggested in mammals that the granulosa cells produce a factor that prevents oocyte maturation (Sato and Koide, 1984; Anderson et al., 1985). The loss of communication between the oocyte and granulosa cell results in a decrease in the amount of this factor transferred to the oocyte, and eventually oocyte maturation is induced (Sirard and Bilodean, 1990).

It is generally considered that the inner cavity of the avian follicle is filled with yolk material and lacks the follicular fluid present in mammalian follicles. The results of this study indicate that fluid accumulated between the surface of the germinal disc and the perivitelline layer. The theca interna was loosened and the intercellular spaces between the granulosa cells were widened when the fluid accumulated. It is therefore probable that the fluid originates from the serum components leaked from the blood capillary sinus in the theca interna and may be similar to the follicular fluid of mammalian follicles. Progesterone, or other steroid hormones secreted by the granulosa cells, stimulates oocyte maturation in lower vertebrates (Haider, 1990). The significance of steroid hormones for oocyte maturation has not been determined in hens. However, if the stimulation by steroid hormones is required for oocyte maturation in hens, the fluid would be important for transport of the hormones from the follicular wall to the oocyte surface. A perivitelline space was observed between the germinal disc and perivitelline layer $1 \mathrm{~h}$ before ovulation. It is considered that this perivitelline space was formed by the spreading of the fluid on the germinal disc.

Marked structural changes were observed in the germinal disc $6 \mathrm{~h}$ before the expected time of ovulation. The elongated membrane-bound vesicles were located in the upper region of the germinal disc before maturation. By $1 \mathrm{~h}$ before ovulation, the membrane-bound vesicles were markedly swollen. Recent studies suggested that maturation-promoting factor is present in an inactive form in the premature oocyte of vertebrates, whereas it is activated under hormonal stimulation at the time of oocyte maturation (Motlik and Kubelka, 1990). It is reported that quail oocytes contain maturation-promoting factor and the activity is markedly increased during oocyte maturation (Mori et al., 1991). The structural changes of the ooplasm responsible for the activation of maturation-promoting factor have not yet been examined. We suggest that the development of the membrane-bound vesicles may be responsible for the activation of maturation-promoting factor, but further studies are required to confirm this.

In summary, it is suggested that a disintegration of the intercellular junctions between the oocyte and granulosa cells and an accumulation of the follicular fluid in the perivitelline space occur at the early stage of oocyte maturation, since the chromosomes at that time are still in the postlampbrush stage of meiosis. The transition of the quail postlampbrush chromosomes into the chromosomes of the first maturation spindle is only observed later, at the moment of the germinal vesicle breakdown (Callebaut, 1973). The marked development of the membrane-bound vesicles in the germinal disc may be related to the activation of factors that promote maturation of the oocyte. However further studies are required to investigate this suggestion.

We wish to thank J. M. Bahr, University of Illinois, for valuable comments and critical reading of the manuscript.

\section{References}

Anderson LD, Stone SL and Channing CP (1985) Influence of hormones on the inhibitory activity of oocyte maturation present in conditioned media of porcine granulosa cells Gamete Research 12 119-130

Calvo FO, Wang S-C and Bahr JM (1981) LH-stimulable adenylyl cyclase activity during the ovulatory cycle in granulosa cells of the three largest follicles and the postovulatory follicle of the domestic hen (Gallus domesticus) Biology of Reproduction 25 805-812

Callebaut M (1973) Correlation between germinal vesicle and oocyte development in the adult Japanese quail (Coturnix coturnix japonica). A cytochemical 
and autoradiographic study Journal of Embryology and Experimental Morphology 29 145-157

Callebaut M (1983) Electron microscope study of ticos $\left({ }^{3} \mathrm{H}\right.$-thymidine incorporating cytoplasmic organelles) in the germinal disc of the postlampbrush oocyte of Japanese quail IRCS Medical Science Clinical Medicine and Surgery 11 491-492

Callebaut $\mathbf{M}$ (1987) Ooplasmic localization and segregation in quail germs: fate of the four ooplasms Archives de Biologie 98 441-473

Doi O, Takai T, Nakamura $T$ and Tanabe $Y$ (1980) Changes in the pituitary and plasma $\mathrm{LH}$, plasma and follicular progesterone and estradiol, and plasma testosterone and estrone concentrations during the ovulatory cycle of the quail (Cotumix cotumix japonica) General and Comparative Endocrinology 41 156-163

Gilula NB, Epstein ML and Beers WH (1978) Cell-to-cell communication and ovulation Journal of Cell Biology 78 58-75

Haider S (1990) Further experimental evidence in support of the involvement of ovarian follicles in oocyte maturation of the Indian catfish, Mystus vittatus General and Comparative Endocrinology 80 80-84

Hyttel P, Callesen H and Greve T (1986) Ultrastructural features of preovulatory oocyte maturation in superovulated cattle Journal of Reproduction and Fertility $76645-656$

Johnson AL and van Tienhoven A (1980) Plasma concentration of six steroids and LH during the ovulatory cycle of the hen, Gallus domesticus Biology of Reproduction 23 386-393

Lindner HR, Tsafriri A, Lieberman ME, Zor U, Koch Y, Bauminger S and Barnea A (1974) Gonadotrophin action on cultured Graafian follicles: induction of maturation division of mammalian oocyte and differentiation of the luteal cell Recent Progress in Hormone Research 30 79-138

Mori M, Yamashita M, Yoshikuni M, Fukada S and Nagahama Y (1991) Maturation-promoting factor and $\mathrm{p} 34^{\text {sid2 } 2}$ kinase during oocyte maturation of the Japanese quail Developmental Biology 146 246-249

Motlik J and Kubelka M (1990) Cell-cycle aspects of growth and maturation of mammalian oocytes Molecular Reproduction and Development 27 366-375
Nishida T, Seki M, Mochizuki K and Seta S (1977) Scanning electron microscopic observations on the microvascular architecture of the ovarian follicles in domestic fowl Japanese Journal of Veterinary Science 39 347-352

Olsen MW (1942) Maturation, fertilization, and early cleavage in the hen's egg. Joumal of Morphology 70 513-533

Olsen MW and Fraps RM (1950) Maturation changes in the hen's ovum Joumal of Experimental Zoology 114 475-489

Patino R and Thomas P (1990) Effects of gonadotropin on ovarian intrafollicular processes during the development of oocyte maturational competence in a teleost, the Atlantic croaker: evidence for two distinct stages of gonadotropic control of final oocyte maturation Biology of Reproduction 43 818-827

Perry MM, Gilbert AB and Evans AJ (1978a) Electron microscope observations on the ovarian follicle of the domestic fowl during the rapid growth phase Joumal of Anatomy 125 481-497

Perry MM, Gilbert AB and Evans AJ (1978b) The structure of the germinal disc region of the hen's ovarian follicle during the rapid growth phase Journal of Anatomy 127 379-392

Phillips RE and Warren DC (1937) Observations concerning the mechanism of ovulation in the fowl Journal of Experimental Zoology 76 117-136

Sato $E$ and Koide SS (1984) A factor from bovine granulosa cells preventing oocyte maturation Differentiation 26 59-62

Sirard MA and Bilodeau S (1990) Granulosa cells inhibit the resumption of meiosis in bovine oocyte in vitro Biology of Reproduction 43 777-783

Tilly JL and Johnson AL (1987) Presence and hormonal control of plasminogen activator in granulosa cells of the domestic hen Biology of Reproduction $\mathbf{3 7}$ 1156-1164

Tsafriri A, Dekel N and Bar-Ami S (1982) The role of oocyte maturation inhibitor in follicular regulation of oocyte maturation Journal of Reproduction and Fertility 64 541-551

Yoshimura $\mathrm{Y}$ and Tamura T (1991) Cyclic adenosine monophosphate promotes the proliferation of the chicken granulosa cells in culture General and Comparative Endocrinology 84 222-227 